\title{
Padrões de Uso de Tocas por Mamíferos em Área de Floresta Estacional Semidecidual no Sul do Brasil
}

\author{
Rômulo da Rosa Silveira ${ }^{1^{*}}$ \& Cristina Vargas Cademartori ${ }^{1,2}$
}

'Laboratório de Conservação e Manejo da Biodiversidade (LabCMBio), Curso de Ciências Biológicas, Universidade La Salle - Unilasalle, Canoas, Rio Grande do Sul, Brasil. *Autor para correspondência: romulor. silveira@gmail. com.

${ }^{2}$ Mestrado em Avaliação de Impactos Ambientais, Universidade La Salle - Unilasalle, Canoas, Rio Grande do Sul, Brasil. E-mail: cristina. cademartori@unilasalle.edu.br.

Abstract. Patterns in use of burrows by mammals in a semi-deciduous season forest area in south Brazil. The aim of this study was to identify and characterize armadillo burrows found in a semideciduous forest remnant employing methods of random-walks, micro-habitat analysis and camera-traps. Most burrows $(60,6 \%)$ were excavated under rocks or tree roots and are associated to different levels of vegetation cover. Three medium and large sized mammals were registered besides small wild rodents, indicating that armadillos may be ecosystem engineers.

Keywords: armadillos, Ecosystem Engineering, microhabitat.

Resumo. O objetivo do estudo foi identificar e caracterizar tocas de tatu encontradas em remanescente de floresta semidecidual empregando os métodos de caminhamento, análise de micro-habitat e armadilhas fotográficas. A maioria das tocas $(60,6 \%)$ foi escavada sob rochas ou raízes de árvores e estão associadas a diferentes níveis de cobertura vegetal. Foram registradas três espécies de mamíferos de médio e grande porte além de pequenos roedores silvestres, indicando que tatus podem ser engenheiros de ecossistemas.

Palavras-chave: Engenharia de Ecossistemas, micro-habitat, tatus.

\section{INTRODUÇÃO}

Os índices de biodiversidade de um ecossistema dependem da variedade de nichos e habitats disponíveis em sua área, uma vez que ambientes heterogêneos oferecem maior variedade de recursos (BEGON, 2010). Embora seja um dos biomas mais ameaçados do mundo, a Mata Atlântica apresenta altos índices de riqueza e endemismo de vertebrados (MYERS et al.,
2000). Inventários realizados na porção austral deste bioma (CADEMARTORI et al., 2002; CHEREM, 2005; SCHEIBLER \& CHRISTOFF, 2007) servem de subsídios para estudos sobre composição da fauna e suas interações com agentes bióticos e abióticos. O entendimento sobre como tais interações contribuem para o arranjo estrutural das comunidades florestais é fundamental para o planejamento de programas de conservação da biodiversidade. 
A grande diversidade de tamanhos e hábitos dos mamíferos é bem conhecida (SILVA, 2014; BECKER \& DALPONTE, 2015), o que permite que os membros deste grupo utilizem os recursos naturais disponíveis de distintas formas, por vezes alterando as dinâmicas da paisagem em que estão inseridos. Por exemplo, espécies de hábitos fossoriais apresentam adaptações específicas para a vida subterrânea e sua atividade pode modificar o ambiente ao seu redor (GARKALIS et al., 2003; SAWYER et al., 2012).

Segundo MCDONOUGH \& Loughry (2008), a maioria dos mamíferos utiliza-se de abrigos para proteção, forrageio e reprodução ao menos durante parte de sua vida; e dos inúmeros tipos de abrigo existentes, poucos oferecem e exigem tanto de seus residentes quanto tocas escavadas no solo (REICHMAN \& SMITH, 1990), tais como as construídas por tatus. Além do mais, alguns estudos indicam a utilização de tocas por diversas espécies da mastofauna, sugerindo que a frequência destas pode ser influenciada pelo padrão de atividade de espécies da família Dasypodidae (DEZBIEZ \& KLUYBER, 2013). A partir disso, alguns estudos investigaram a preferência de habitat por tatus (ANACLETO \& DINIZ-FILHO, 2008; MCDONOUgh et al., 2008); porém, existem poucas informações no que se refere às características do entorno de suas tocas, o micro-habitat. Tais características - padrões de micro-habitat - podem sinalizar áreas potenciais para a ocorrência de tatus, já que a ocupação do espaço é resultado da seleção de características ambientais.
Considerando que as espécies envolvidas na construção de tocas de médio porte compreendem majoritariamente a família Dasypodidae (tatus), pergunta-se: a) há características ambientais preferenciais que determinam a escolha do local onde este tipo de abrigo será construído? b) o arranjo das tocas em um ecossistema influencia a presença e a frequência de algum outro grupo de mamíferos, tais como carnívoros ou roedores? c) é possível identificar a espécie de tatu vinculada à determinada toca e reconhecer, ainda, se está sendo ocupada (ativa) ou se está desocupada (inativa)? Este trabalho, portanto, tem como objetivo geral contribuir para o conhecimento sobre a utilização de tocas escavadas no solo por mamíferos de pequeno e médio porte em ambiente florestal. Deste modo, buscou-se investigar características de micro-habitat que possam estar relacionadas à construção de tocas por espécies da família Dasypodidae (tatus), e verificar a presença de mamíferos de pequeno e médio porte no entorno das tocas monitoradas.

\section{Material e Métodos}

\section{ÁREA DE ESTUDO}

O presente estudo foi realizado no Morro do Coco, área localizada no município de Viamão, Rio Grande do Sul (Figura 1). Assim como outros morros distribuídos na região, o Morro do Coco está inserido no Escudo Sul-Riograndense e caracterizado por solo granítico; sua altitude não ultrapassa os 136 metros (BACKES, 2001). A área abrange um fragmento de aproximadamente 


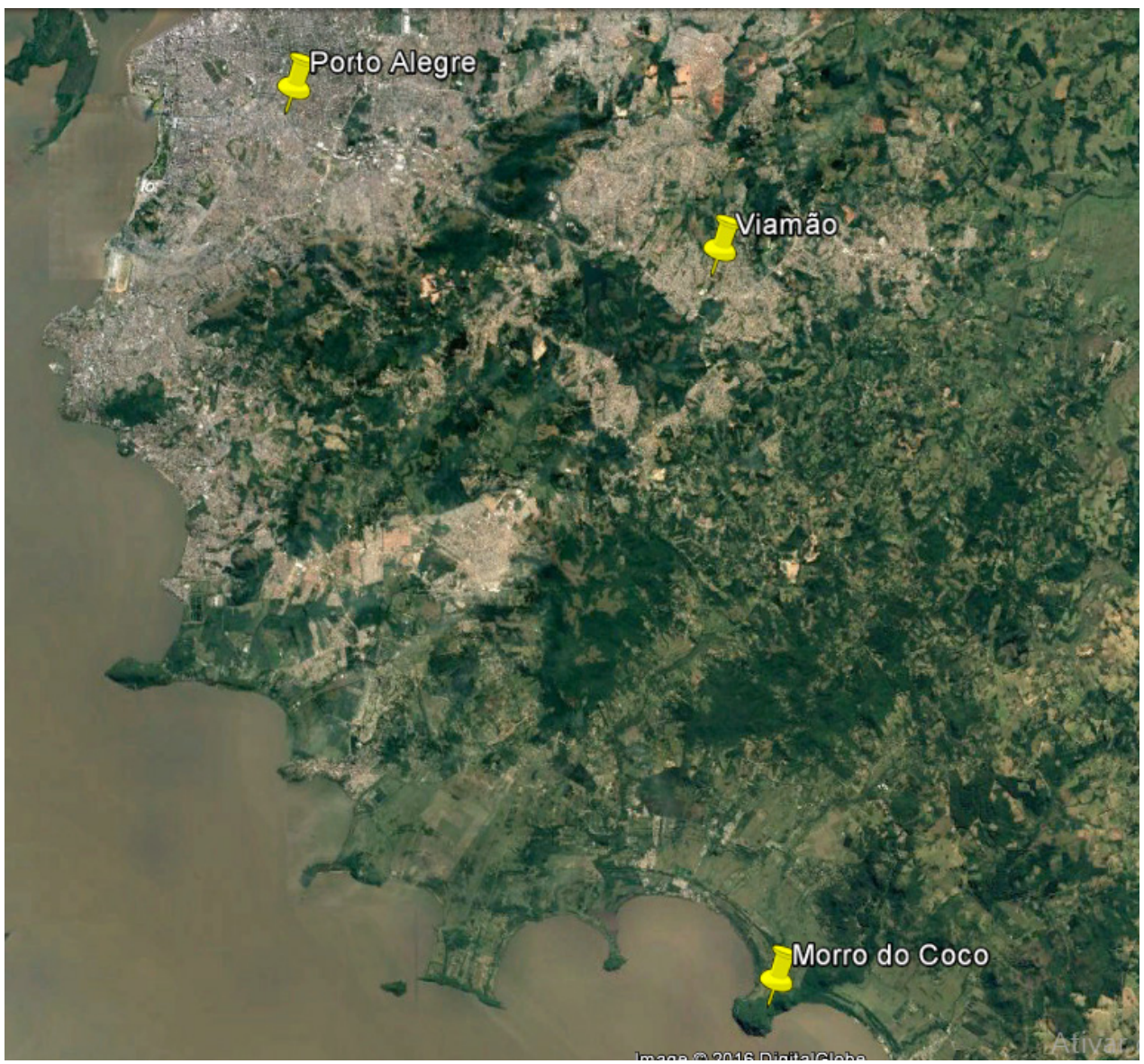

Figura 1. Área de estudo, localizada no município de Viamão, RS, às margens do lago Guaíba, formando a enseada de Itapuã (adaptado de Google Earth, 2016).

142 hectares cobertos por Floresta Estacional Semidecidual em estágio avançado de sucessão ecológica (PIRES \& CADEMARTORI, 2012). A porção sul da área de estudo é delimitada pelo lago Guaíba.

\section{DELINEAMENTO AMOSTRAL}

O período de amostragem compreendeu os meses de maio a novembro de 2016, contando com uma a duas expedições ao mês. Cada expedição teve duração de três dias, totalizando 33 dias de esforço amostral. Os métodos empregados consistiram em análise de micro-habitat, caminhamento (random-walks) e armadilhas fotográficas.

\section{CARACTERIZAÇÃO DAS TOCAS}

Para a localização das tocas, foram percorridas três transecções de 80 a $200 \mathrm{~m}$ de extensão em diferentes trechos da área de estudo, totalizando $400 \mathrm{~m}$ de caminhada (Figura 2 ). As tocas foram classificadas de acordo com os elementos estruturais presentes em sua entrada, tais como raízes, árvores, pedras ou troncos 


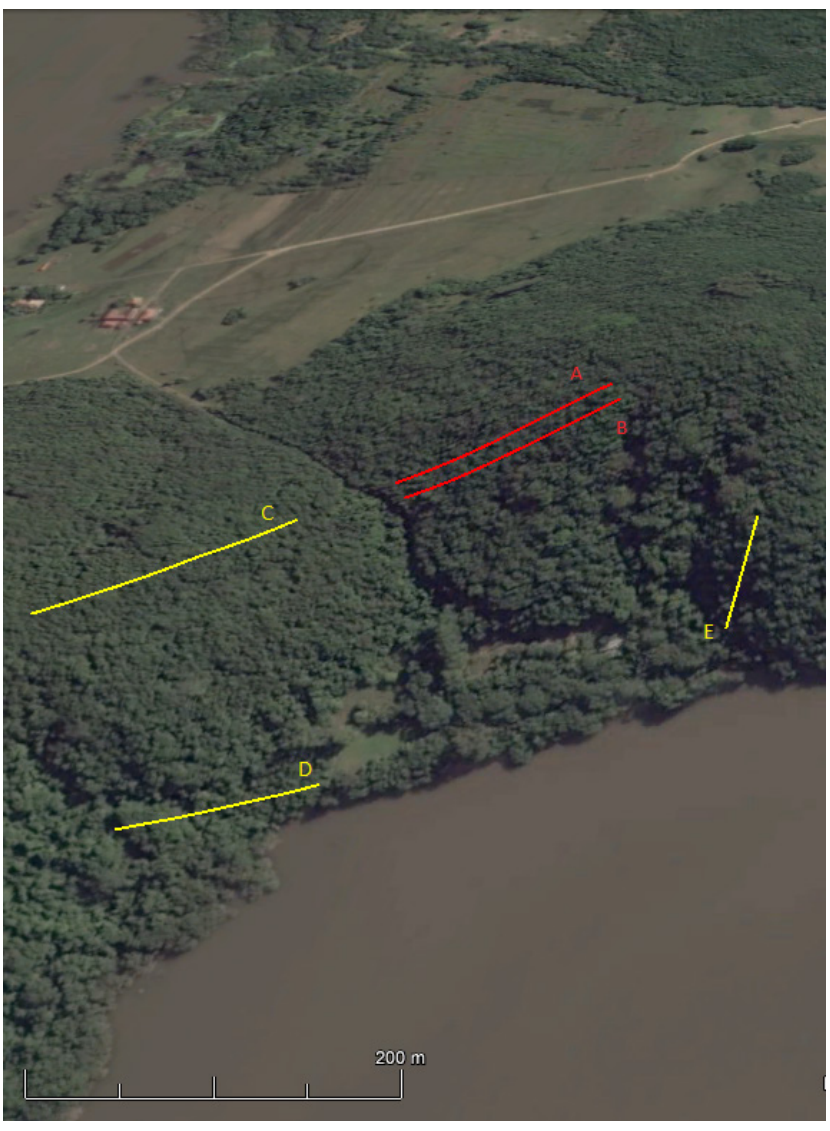

Figura 2. Transecções delimitadas para o mapeamento das tocas utilizadas por mamíferos em remanescente de Floresta Estacional Semidecidual. A e B: transecções de $180 \mathrm{~m}$ de extensão com distribuição equidistante das estações de micro-habitat avaliadas. C, D e E: transecções de 80 a 200 m de extensão percorridas através do método de caminhamento (adaptado de Google Earth, 2016).

caídos; na ausência de um destes elementos, a toca foi classificada como "escavada diretamente no solo".

\section{ANÁlise de Micro-HABitAT}

A análise de micro-habitat compreendeu tocas encontradas ao longo de duas transecções paralelas de 180 metros de comprimento na encosta do morro, onde foram definidas 40 estações de amostragem equidistantes 20 metros, dentro das quais realizou-se a busca ativa de tocas escavadas no solo. A delimitação das estações levou em conta a área de vida e forrageio de $D$. novemcinctus (LOUGHRY \& MCDONOUgh, 1998) e cada uma compreendeu uma área circular de $314 \mathrm{~m}^{2}$, calculada a partir da fórmula $A=\pi r^{2}$, sendo $r=10$. Para selecionar as amostras de forma aleatória, foi realizado o sorteio das estações de trabalho, excluindo-se repetições. As tocas encontradas em cada uma das estações sorteadas foram demarcadas e classificadas de acordo com os tipos descritos na seção anterior. Além disso, as dimensões (altura e largura) de cada toca foram mensuradas e comparadas a estudos semelhantes a fim de confirmar a espécie de tatu responsável pela sua construção.

Visando representar as características ambientais das tocas demarcadas nas estações de amostragem selecionadas, foram mensuradas características de micro-hábitat em um raio de três metros a partir de cada toca (adaptado de LIMA et al., 2010), de forma que fosse possível verificar a existência ou não de padrões ecológicos na área amostrada. A correlação entre as variáveis abaixo relacionadas foi analisada através de PCA (Análise de Componentes Principais), utilizando-se o software estatístico PAST v. 3.14. A análise compreendeu oito variáveis a seguir discriminadas: 1) jovens e brotos, que representaram o número de plantas com diâmetro a altura do peito (DAP) inferior a $10 \mathrm{~cm}$, bem como mudas e brotos; 2) rochas, ou seja, o número de rochas graníticas (tamanhos 
variados) no entorno; 3) árvores $10-20 \mathrm{~cm}$, que consistiu no número de indivíduos arbóreos com DAP entre 10 e $20 \mathrm{~cm}$; 4) árvores $20-30 \mathrm{~cm}$, o número de indivíduos arbóreos com DAP entre 20 e $30 \mathrm{~cm}$; 5) árvores $30-50 \mathrm{~cm}$, o número de indivíduos arbóreos com DAP entre 30 e 50 cm; 6) árvores acima de $50 \mathrm{~cm}$, o número de indivíduos arbóreos com DAP superior a $50 \mathrm{~cm}$ em uma distância de dois a três metros da toca; 7) peso da serapilheira, o peso inicial da serapilheira coletada em um raio de $50 \mathrm{~cm}$ da entrada e 8) umidade da serapilheira, calculada a partir da fórmula 100-peso inicial/ peso final (o peso final foi obtido após secagem do material em estufa a 90 ㄷ em um período de cinco dias).

\section{ATIVIDADE DE MAMÍFEROS}

Ao longo do estudo foram instaladas duas armadilhas fotográficas tipo Bushnell em frente a tocas que mostrassem evidências de atividade, tais como entrada desobstruída, pegadas, fezes e fuçadas. Este método visou identificar as espécies de mamíferos que estariam utilizando determinada toca no período da amostragem e totalizou 32 minutos de filmagem com um esforço de 119 armadilhas-dia.

\section{RESULTADOS E Discussão}

Foram contabilizadas 23 tocas pelo método de caminhamento, classificadas qualitativamente quanto aos elementos estruturais de sua entrada, e outras 10 distribuídas nas estações de amostragem e análise de microhabitat, somando, ao todo, 33 tocas (Tabela 1). Estas foram atribuídas a Dasypus novemcinctus (Linnaeus, 1758), única espécie de tatu registrada ao longo das amostragens. Embora haja registros de outras espécies que habitam tocas na área de estudo, tais como Dasyprocta azarae (Lichtenstein, 1823) (PIRES \& CADEMARTORI, 2012), as dimensões encontradas são similares àquelas relatadas em outros estudos para $D$. novemcinctus (Figura 3).

Os tipos mais frequentes de tocas foram as escavadas sob pedras ou raízes, seguidas das tocas escavadas diretamente no solo (Tabela 1). Contudo, 76\% apresentaram algum elemento de obstrução em sua entrada. Este mesmo fenômeno é relatado por SAWYER et al. (2012), indicando que talvez haja preferência por locais que favoreçam a obstrução da entrada, proporcionando maior

Tabela 1. Frequência de tocas utilizadas por mamíferos em área de Floresta Estacional Semidecidual, de acordo com o tipo de estrutura identificado.

Tipo de toca

\begin{tabular}{ccc}
\hline Sob rocha & 10 & 30,30 \\
Sob raiz & 10 & 30,30 \\
No solo & 8 & 24,24 \\
Sob árvore & 4 & 12,12 \\
Sob tronco caído & 1 & 3,04 \\
Total & 33 & 100 \\
\hline
\end{tabular}




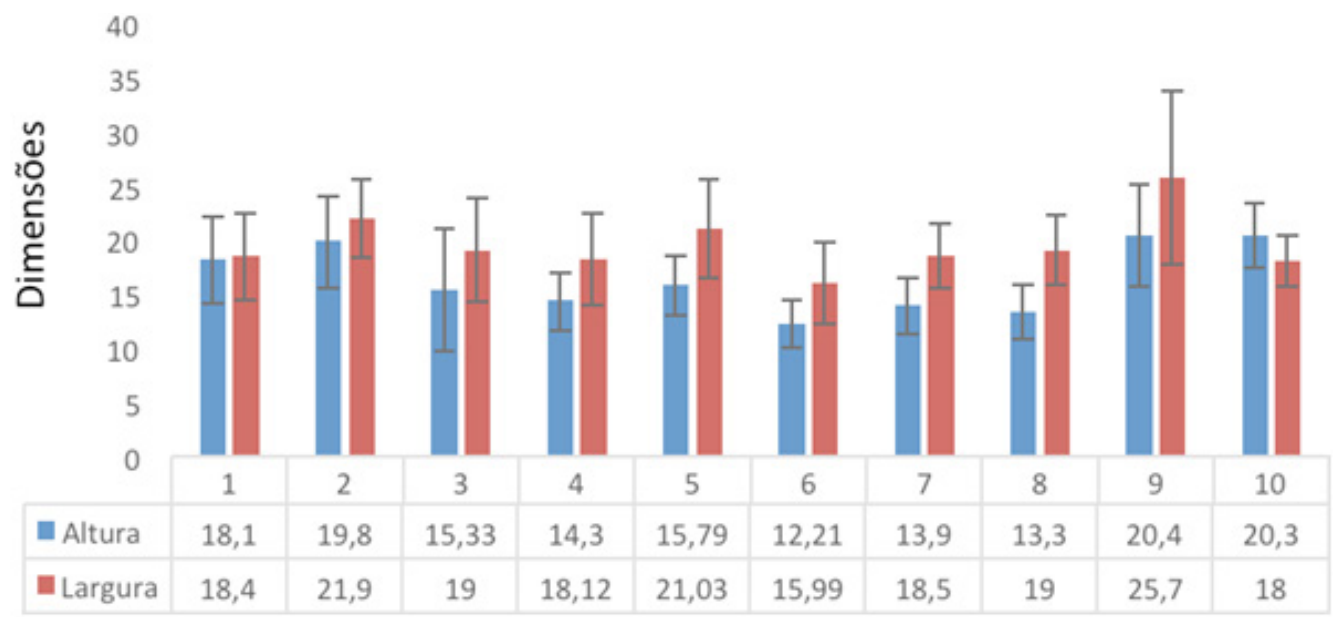

Figura 3. Média das dimensões (altura e largura em $\mathrm{cm}$ ) de tocas usadas por mamíferos, obtidas por distintos autores. As colunas correspondem à média das variáveis com seus respectivos desvios-padrão. 1 - Presente estudo; 2 - ZIMMERMAN (1990); 3 a 6 - MCDONOUgh et al. (2000); 7 e 8-PLATT (2004); 9 - ANACLETO \& DinIZ-FILHo (2008); 10 -SAWYER et al. (2012).

proteção e exigindo menor gasto energético na explicam em torno de $60 \%$ da variação dos dados construção da toca.

\section{CARACTERÍSTICAS DE MICRO-HABITAT}

(Figura 4). O eixo X (Componente 1) apresenta valores mais altos no que se refere à quantidade de árvores e serapilheira, ao passo que a presença

Os dois principais componentes da PCA de pedras e brotos apresenta valores mais baixos.

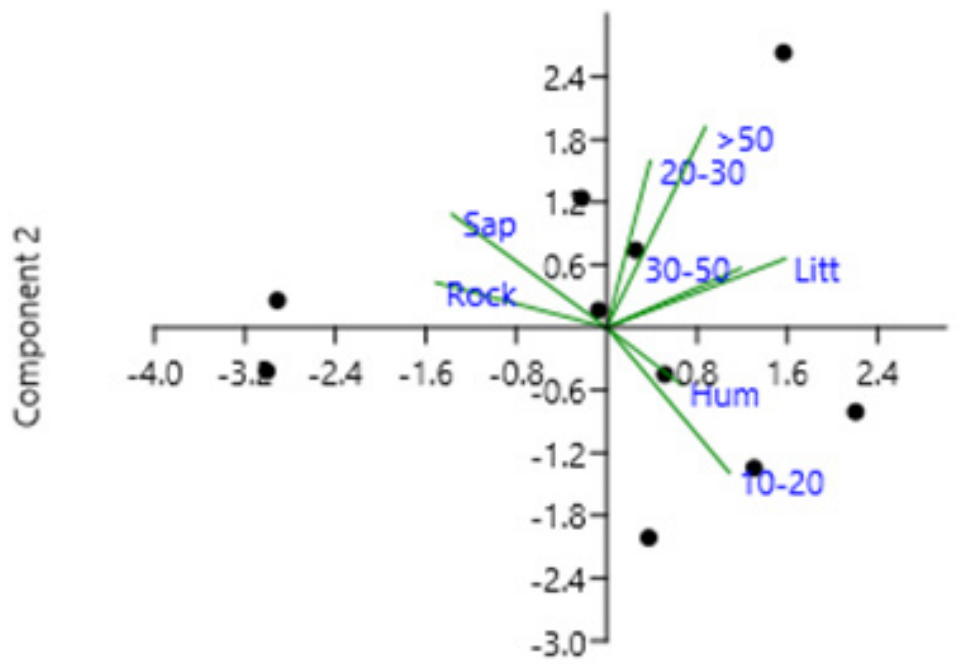

\section{Component 1}

Figura 4. Análise de Componentes Principais indicando as características responsáveis pela maior parte da variação encontrada em tocas usadas por mamíferos em Floresta Estacional Semidecidual. Abreviações: Sap = Jovens e brotos, Rock = Rochas, Litt $=$ Peso da serapilheira, Hum = Umidade da serapilheira, 10-20 = Indivíduos arbóreos com DAP entre 10 e $20 \mathrm{~cm}, 20-30$ = Indivíduos arbóreos com DAP entre 20 e $30 \mathrm{~cm}, 30-50$ = Indivíduos arbóreos com DAP entre 30 e 50 cm, $>50$ = Indivíduos arbóreos com DAP igual ou superior a $50 \mathrm{~cm}$. 
O eixo Y (Componente 2) indica a predominância de indivíduos arbóreos com DAP superior a 20 $\mathrm{cm}$, em contraste com indivíduos com DAP entre 10-20 cm. De modo geral, a PCA indica que a variação das tocas está relacionada a diferentes níveis de cobertura vegetal e a porções da mata em estádios sucessionais mais avançados.

\section{ATIVIDADE DE MAMÍFEROS}

Registrou-se a presença de ao menos quatro espécies de mamíferos no entorno das tocas (Tabela 2). $O$ tatu-galinha, D. novemcinctus, foi a espécie mais frequente, registrando-se atividades que envolviam a manutenção da toca, como a remoção do excesso de folhiço e a escavação de entradas adicionais em tocas já existentes. Pequenos roedores foram registrados utilizando as tocas como abrigo, enquanto Leopardus guttulus (Hensel, 1872) e Cerdocyon thous (Linnaeus, 1766) foram avistados vasculhando as entradas e o entorno das tocas, possivelmente à procura de presas (Figura 5). De fato, o registro destes carnívoros ocorreu principalmente após a passagem de tatus ou roedores pelo local, confirmando o que diz Mohammadi (2010) a respeito da influência

Tabela 2. Espécies de mamíferos e o tipo de toca em que foram registradas em remanescente de Floresta Estacional Semidecidual. $\operatorname{Tr}=$ Tronco caído; Roc = Sob rocha.

\begin{tabular}{cl}
\hline Espécie & Tipo de Toca \\
\hline Cerdocyon thous (Linnaeus, 1766) & $\mathrm{Tc}, \mathrm{Roc}$ \\
Dasypus novemcinctus (Linnaeus, 1758) & $\mathrm{Tr}, \mathrm{Roc}$ \\
Leopardus guttulus (Hensel, 1872) & $\mathrm{Tr}$ \\
Pequenos roedores & $\mathrm{Tr}, \mathrm{Roc}$ \\
\hline
\end{tabular}

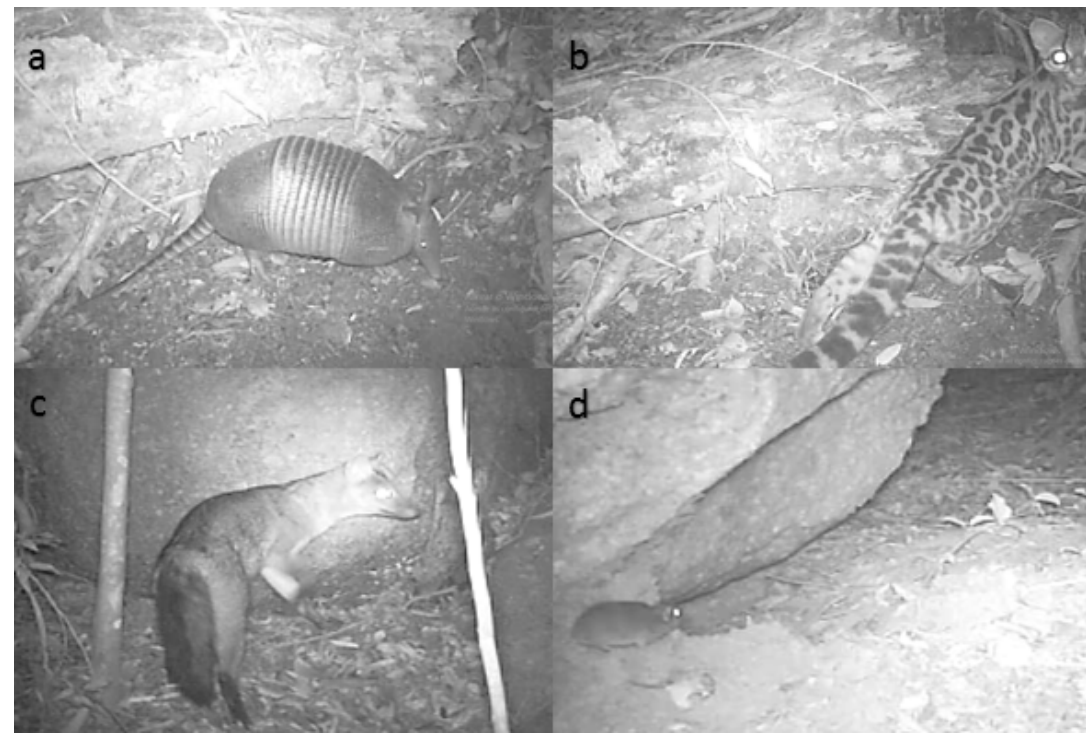

Figura 5. Mamíferos de pequeno e médio porte registrados na entrada de toca em Floresta Estacional Semidecidual no Morro do Coco, Viamão, RS: a) Dasypus novemcinctus, b) Leopardus guttulus, c) Cerdocyon thous e d) pequenos roedores silvestres. 
de presas sobre a frequência de predadoresem dada área.

Muitos autores consideram espécies escavadoras como engenheiros de ecossistemas (DEZBIEZ \& KLUYBER, 2013; LAMBERTo et al., 2014). Este conceito, introduzido por JONES et al. (1994), refere-se às espécies cujas atividades modificam as características físico-químicas do ambiente em que estão inseridas. Embora o autor não tenha se preocupado com a magnitude destes efeitos, REICHMANN \& SEABLOOM (2002) discorrem sobre a intensidade dos efeitos da atividade escavadora de roedores da família Geomyidae sobre aspectos físicos dos ecossistemas em que ocorrem. WILBY (2002), porém, sugere que a engenharia de ecossistemas envolve outros fatores além dos físico-químicos, uma vez que influencia e altera também os fluxos de energia do ecossistema. Assim sendo, as interações tróficas mediadas pelas alterações físicas devem ser levadas em conta a fim de estabelecer o valor desses efeitos. Este seria o caso de $D$. novemcinctus, que pode facilitar o encontro de presas e predadores (roedores $\mathrm{x}$ carnívoros) sem necessariamente participar da interação, caracterizando uma relação assimétrica (os tatus afetam outras espécies, mas não são afetados por elas).

\section{CONCLUSÕES}

A caracterização das tocas possibilitou identificar Dasypus novemcinctus como a espécie escavadora, através das dimensões encontradas nas entradas, além de apontar sua preferência por escavar tocas sob elementos pré-existentes no solo, como rochas e raízes. A análise de microhabitat, por sua vez, indicou certa preferência por locais com maior cobertura vegetal a áreas mais abertas (clareiras, por exemplo).

A ocorrência de espécies comensais no entorno das tocas sugere que o tatu-galinha pode atuar como engenheiro de ecossistemas, proporcionando vias alternativas para os fluxos de matéria e energia, neste caso modificando relações de predação entre predadores da ordem Carnivora e pequenos roedores silvestres na medida em que cria novos hábitats.

Os resultados obtidos apontam a necessidade de investigar de forma intensiva a influência da família Dasypodidae em ambientes florestais, uma vez que engenheiros de ecossistemas como os tatus contribuem para a heterogeneidade do ecossistema e influenciam a distribuição e abundância de outros organismos.

\section{REFERÊNCIAS BIBLIOGRÁFICAS}

AnACLETO, T.C.S; Diniz-Filho, J.A.F. 2008. Efeito da alteração antrópica do cerrado sobre a comunidade de tatus (Mammalia, Cingulata, Dasypodidae), pp. 55-67. In: Reis, N.R.; Peracchi, A. \& SAntos, G.A.S.D. (org.). Ecologia de mamíferos. Londrina, Technical Books. 167p.

BACKES, A. 2001. Ecologia da floresta latifoliada do Morro do Coco, Viamão, Rio Grande do Sul. II - Produção de serapilheira, de $\mathrm{CO}_{2^{\prime}}$ pelo solo e decomposição de celulose. Iheringia - Série Botânica 55: 3-21. 
BECKER, M. \& DALPONTE, J.C. 2015. Rastros de mamíferos silvestres brasileiros. 3. ed. Rio de Janeiro, Technical Books. 166p.

CHEREM, J.J. 2005. Registros de mamíferos não voadores em estudos de avaliação ambiental no sul do Brasil. Biotemas 18 (2): 169-202.

Desbiez, A.L.J. \& KLUyBeR, D. 2013. The Role of Giant Armadillos (Priodontes maximus) as Physical Ecossystem Engineers. Biotropica 45 (5): 537-540.

GARKAKLIS, M.J.; BRAdLEY, J.S. \& WoOlLeR, R.D. 2003. The relationship between animal foraging and nutrient patchiness in southwest Australian woodland soils. Soil Research 41 (4): 665-673.

HAMMeR, O.; HARPER, D.A.T. \& RYAN, P.D. 2001. PAST: Paleontological statistics software package for education and data analysis. Palaeontologia Electronica 4 (1): 9-12.

JONES, C.G.; LAWTON, J.H. \& SHACHAK, M. 1994. Organisms as Ecossystem Engeneers. Oikos 63 (3): 373-386.

LAMberto, J.F.; Strona, A.L.S.; Cardoso, S.T. \& LEINER, L.O. 2014. O papel de Clyomys laticeps (rodentia: Echymidae) como engenheiro do ecossistema no cerrado. In: VII CONGRESSO BRASILEIRO DE MAstozoologiA, Gramado, Brasil. pp. 399.

LimA, D.O.; Azambuja, B.O.; CAMilotTi, V.L. \& CÁCERES, N.C. 2010. Small mammal community structure and micro-habitat use in the austral boundary of the Atlantic Forest. Zoologia 27 (1): 99-105.

LOUghrY, W.J. \& MCDONOUGH, C.M. 1998. Spatial Patterns in a Population of Nine-Banded Armadillos (Dasypus novemcinctus).

Mcdonough, C.M.; Delaney, M.J.; LE, P.Q.; BLAKCMOORE, M.S. \& LOUGHRY, W.J. 2000. Burrow characteristics and habitat associations of armadillos in Brazil and the United States of America. Revista de Biologia Tropical 48 (1): 109-120.

MoHAMmAdI, S. 2010. Microhabitat Selection by Small Mammals. Advances in Biological Research 4 (5): 283-287.

Myers, N.; Mittermeier, R.A.; FonsecA, G.A.B. \& KENT, J. 2000. Biodiversity hotspots for conservation priorities. Nature 403: 853858.

PIRES, D.P.S. \& CAdEMARTORI, C.V. 2012. Medium and large sized mammals of a semideciduous forest remnant in southern Brazil. Biota Neotropica 12 (3): 239-245.

SILVA, F. 2014. Mamíferos silvestres: Rio Grande do Sul. 3. ed. Porto Alegre, Via Sapiens. $308 p$.

TOWNSEND, C.R.; Begon, M.B. \& HARPER, J.L. 2010. Fundamentos em Ecologia. 3. ed. Porto Alegre, Artmed. 576p.

ZIMMERMAN, J.W. 1990. Burrow characteristics of the nine-banded armadillo, Dasypus 
70. SILVEIRA \& CADEMARTORI

novemcinctus. The Southwestern

Naturalist 35 (2): 226-227.
Recebido: 22/04/2017

Revisado: 13/05/2017

Aceito: 27/05/2017 